discriminated between high and low risk for RP among patients with high SJC $(>5)$ or high DAS28-CRP, with PPV as high as $57 \%$.

Conclusions: High MBDA scores were associated with increased risk for RP in 6 study cohorts, including patients treated with csDMARDs, TNFi and abatacept. Based on high NPVs ( $\geq 93 \%$ ), the MBDA score used alone had clinical value for identifying patients with little or no risk of RP. Combining the MBDA score with clinical measures yielded PPVs approaching $60 \%$, suggesting that biomarkers can help stratify patients by their risk for RP.

Disclosure of Interest: J. Curtis Grant/research support from: Crescendo Bioscience Inc., Consultant for: Crescendo Bioscience Inc., C. Brahe: None declared, M. Ostergaard Grant/research support from: AbbVie, BMS, Boehringer-Ingelheim, Eli Lilly, Janssen, Merck, Pfizer, Roche, UCB, Celgene, Sanofi, Regeneron, Novartis, T Jensen, M. Hetland Grant/research support from: AbbVie, BMS, MSD, Pfizer, Crescendo Bioscience Inc., UCB, Eli Lilly, Speakers bureau: Orion, K. Hambardzumyan: None declared, S. Saevarsdottir: None declared, X. Wang Shareholder of: Myriad Genetics, Inc., Employee of: Crescendo Bioscience Inc., E. Sasso Shareholder of: Myriad Genetics, Inc., Employee of: Crescendo Bioscience Inc., T. Huizinga Consultant for: Merck, UCB, Bristol Myers Squibb, Biotest AG, Pfizer, GSK, Novartis, Roche, Sanofi-Aventis, Abbott, Crescendo Bioscience Inc., Nycomed, Boeringher, Takeda, Zydus, Epirus, Eli Lilly DOI: 10.1136/annrheumdis-2017-eular.5557

\section{THU0092 CHORONIC PAIN INCREASES INDEPENDENT OF THE DISEASE ACTIVITY AND DEPRESSION IN FEMALES WITH RHEUMATOID ARTHRITIS}

F.G. Yurdakul, A. Kılıçarslan, H. Bodur. Department of Physical Medicine and Rehabilitation, Ankara Numune Training and Research Hospital, Ankara, Turkey

Background: Chronic pain is a key component of rheumatoid arthritis (RA). Although pain is reduced with the control of inflammation at the first years of the disease, pain increases over time with different pathways just as central sensitization. Fatigue, sleep problems and depressive symptoms with chronic pain are common problems in patients with RA $(1,2)$.

Objectives: We aimed to investigate the frequency of widespread pain, sleep disorders, fatigue, and depressive symptoms in RA patients. Furthermore discrepancy of these symptoms and disorders were analyzed between female and male RA patients.

Methods: One hundred and sixty one RA patients (female: 119, male: 42) and 68 healthy controls (female: 52 , male: 16 ) were enrolled in the study. Widespread pain index (WPI) with nineteen body parts that was identified by 2010 fibromyalgia diagnostic criteria was interrogated. Pain visual analog scale (VAS), Health Assessment Questionnaire (HAQ), Physician global assessment (PhGA), Fatigue severity scale (FSS), Pittsburgh sleep quality index (PSQI), Beck depression inventory (BDI) were evaluated in both RA patients and healthy controls. Morning stiffness (MS), Rheumatoid arthritis quality of life (RAQOL) and disease activity score 28 (DAS28) were assessed in RA patients. Data were analyzed in female and male RA patients.

Results: The mean PhGA, HAQ, BDI, FSS, WPI values of RA patients were worse than healthy controls $(p=0.012,0.000,0.008,0.033,0.044$ respectively). There was no difference between RA and healthy controls in terms of sleep disorders. The mean age, disease duration, MS, swollen joint count, C-reactive protein, PhGA and BDI were similar in female and male RA patients. WPI, VAS pain, tender joint count, HAQ, RAQOL, FSS, PSQI, and DAS 28 were higher in females (Table 1).

Table 1. Clinical features in female and male RA patients

\begin{tabular}{lccc}
\hline & Female RA patients $(\mathrm{n}=119)$ & Male RA patients $(\mathrm{n}=42)$ & $\mathrm{p}$ \\
\hline Widespread pain index & $4.70 \pm 4.97$ & $2.65 \pm 3.99$ & $0.04^{\star}$ \\
PhGA & $2.87 \pm 2.16$ & $2.09 \pm 1.63$ & 0.08 \\
DAS28 & $3.39 \pm 1.39$ & $2.68 \pm 1.11$ & $0.01^{\star}$ \\
CRP & $10.77 \pm 13.62$ & $12.59 \pm 14.20$ & 0.24 \\
Tender Joint Count & $10.92 \pm 13.47$ & $6.78 \pm 13.09$ & $0.03^{*}$ \\
Swollen Joint Count & $0.88 \pm 2.67$ & $0.31 \pm 0.69$ & 0.690 \\
HAQ & $1.06 \pm 0.83$ & $0.68 \pm 0.66$ & $0.04^{*}$ \\
RAQOL & $14.34 \pm 10.06$ & $7.59 \pm 8.83$ & $0.00^{*}$ \\
FSS & $35.57 \pm 19.24$ & $22.21 \pm 14.41$ & $0.00^{*}$ \\
PSQI & $8.02 \pm 5.58$ & $5.00 \pm 4.20$ & $0.00^{*}$ \\
BDI & $15.71 \pm 13.34$ & $11.28 \pm 12.56$ & 0.06 \\
\hline
\end{tabular}

PhGA: Physician global assessment, DAS: Disease activity score, CRP: C Reactive protein, HAQ: Health Assessment Questionnaire, RAQOL: Rheumatoid arthritis quality of life, FSS: Fatigue severity scale, PSQI: Pittsburgh sleep quality index, BDI: Beck depression inventory.

Conclusions: RA is a disease that increases fatigue, depressive symptoms and widespread pain.DAS 28 scores were higher due to the increased pain scores and tender joint count that are subjective parameters in female RA patients. Pain scores in females are significantly higher than in males, and pain exacerbated by central sensitization pathway in women may lead to sleep disorders and fatigue, but not increase depressive symptoms.

\section{References:}

[1] The role of central nervous system in the generation and main tance of chronic pain in rheumatoid arthritis, osteoarthritis and fibromyalgia. Lee YC, Nassikas NJ, Clauw DJ. Arthritis Research and Therapy (2011) 13:211-221.
[2] Fatigue in patients with rheumatic diseases. Dupond JL. Joint Bone Spine (2011). 78:156-160

Disclosure of Interest: None declared

DOI: 10.1136/annrheumdis-2017-eular.5846

\section{THU0093 PERSISTENCE OF POWER DOPPLER ULTRASOUND-DETECTED RESIDUAL SYNOVITIS IN CONSECUTIVE ULTRASOUND EXAMINATIONS IN RHEUMATOID ARTHRITIS PATIENTS IN CLINICAL REMISSION PREDICTS UNFAVORABLE OUTCOME OVER ONE YEAR}

G. Mouterde ${ }^{1}$, C. Lukas ${ }^{1}$, N. Filippi ${ }^{1}$, G. Marin ${ }^{2}$, N. Molinari ${ }^{2}$, J. Morel $^{1}$, B. Combe ${ }^{1}$. ${ }^{1}$ Rheumatology department; ${ }^{2}$ Department of Statistics, Lapeyronie hospital, Montpellier, France

Background: Some studies revealed an association of Power Doppler (PD) ultrasound (US)-detected residual synovitis (PDUSS) and risk of relapse and radiographic progression ( $R P$ ), in individual patients in rheumatoid arthritis (RA). However, the longitudinal relationship between clinical remission and repeated US residual lesions during follow-up is not so well-known.

Objectives: The aim of this study was to evaluate the ability of PDUSS in consecutive examinations to predict unfavorable outcome (i.e. clinical relapse or $\mathrm{RP})$ at 1 year.

Methods: RA patients $\geq 18$ years fulfilling 2010 ACR-EULAR criteria, treated with synthetic or biologic (b) DMARDs and in clinical remission (DAS28-ESR $<2.6$ and no clinically active synovitis) for less than 6 months, were included in the longitudinal prospective SONORE study (ClinicalTrials.gov identifier: NCT02618954). Clinical and biological characteristics of patients were collected at baseline, and every 3 months during 1 year. RA treatment had to be stable during follow-up. A standard US examination on 40 joints for the presence of synovial hypertrophy and PD signal was performed by an independent investigator blinded to clinical and radiographic data at each visit during 1 year. Presence of US synovitis was defined by a PD signal $>1$ in at least one joint. Radiographs of hands, wrists and feet were scored at baseline and 1 year. Outcome measures: $\mathrm{RP}$ was defined by an increase $\geq 1$ point of the modified total Sharp score. A relapse was defined by a DAS28 $>3.2$ at $\geq 1$ follow-up visits AND a change of DMARDs, excluding change due to safety issues; or an increase in the DMARD or Corticosteroid (CS) dosage ( $>5 \mathrm{mg} / \mathrm{d}$ ). Baseline variables, including PDUSS and its persistence during the follow-up, were assessed for their association with time to progression to unfavorable outcome using univariate then stepwise multivariate Cox regression analyses to obtain adjusted HRs.

Results: The 115 included patients had a mean (SD) age of $58.9( \pm 12.8)$ years, mean disease duration of $9.3( \pm 9.3)$ years, a mean duration of remission of 2.1 $( \pm 2.3)$ months. $74.8 \%$ were female, $79.1 \%$ of the patients were anti-CCP positive, $51.4 \%$ had erosive disease. The mean DAS28-ESR was $2.03( \pm 0.63) .59 .2 \%$ received methotrexate, $59.9 \%$ bDMARD and $11.7 \%$ CS. PDUSS was detected in $\geq 1$ joint in 75 patients $(72.1 \%)$ at baseline. $41 / 75(54.7 \%)$ had persistence of at least one PDUSS during the follow-up. 26 (23.2\%) had a relapse (after a mean duration of $9.1( \pm 2.6)$ months) or a RP at 1 year. In multivariate analysis, persistence of at least one PDUSS during the follow-up ( $\mathrm{HR}=5.24$ [1.74-22.5], $\mathrm{p}=0.009)$ and baseline number of tender joints ( $\mathrm{HR}=1.32$ [0.95-1.68], $p=0.052)$ were predictors of relapse or RP at 1 year. Duration of remission, other baseline US findings including baseline PDUSS, autoantibodies, and erosive disease had no additional predictive value.

Conclusions: Persistence of a PDUSS during the follow-up, rather than baseline PDUSS, predicts unfavorable outcome at 1 year in RA patients in clinical remission. This suggests that initial US findings are not sufficient to justify therapeutic change, but that the persistence of a residual PDUSS requires careful follow-up, and might even potentially merit strategy adaptation.

Disclosure of Interest: None declared

DOI: 10.1136/annrheumdis-2017-eular.5479

\section{THU0094 FACTORS ASSOCIATED WITH RADIOGRAPHIC REMISSION (RR) IN PATIENTS WITH RHEUMATOID ARTHRITIS (RA) WHILE NON-BIOLOGICALDISEASE MODIFYING ANTI-RHEUMATIC DRUGS (DMARDS) USING}

O. laremenko, G. Mykytenko. Internal Diseases at Stomatological Faculty, O.O. Bogomolets National Medical University, Kyiv, Ukraine

Objectives: To assess the factors associated with RR achievement in patients (pts) with RA while non-biological DMARDs using.

Methods: A cohort of 174 pts with RA (50.6\% with early RA) was prospectively assessed at baseline and 2 years by the Disease Activity Score (DAS28) and the Sharp-van der Heijde Score (SHS). Mean age at inclusion was 52.0 \pm 0.91 yrs, disease duration $-51.3 \pm 4.82$ month. $82.7 \%$ of the pts were women; $62.6 \%$ were positive for rheumatoid factor (RF) and $75.9 \%$ - for antibodies against cyclic citrullinated peptides (aCCP). Pts were treated with methotrexate (MTX) (mean dose $-11.6 \pm 0.29 \mathrm{mg} / \mathrm{w}, \mathrm{n}=157)$, leflunomide (LF) $(19.2 \pm 0.28 \mathrm{mg} / \mathrm{d}, \mathrm{n}=95)$, sulfasalazine (SS) $(2 \mathrm{~g} / \mathrm{d}, \mathrm{n}=76)$ or combination of DMARDs (CD) $(\mathrm{n}=74)$. After 2 yrs of DMARDs therapy 41 pts $(23.5 \%)$ reached RR $(\Delta S H S \leq 0.5)$. No one pts using low dose of MTX (7.5 mg/week) achieved remission so they were excluded from further analysis. According to RR achievement in 2 yrs pts were divided into 
2 groups (41and 92 pts who achieved RR and didn't respectively). After 2 yrs the factors associated with RR achievement in pts with RA while non-biological DMARDs using were assessed.

Results: Before study, pts in both groups were comparable by all demographic, clinical and X-ray characteristics, frequency of DMARDs prescribed. They differed only in frequency of RF-positivity (39.0 and 57.6\% respectively in pts achieved $\mathrm{RR}$ and didn't, $\mathrm{p}<0.05$ ), aCCP-positivity ( 21.7 and $73.3 \%$ respectively, $\mathrm{p}<0.001$ ) and aCCP level $(6.06 \pm 0.88$ and $105.3 \pm 22.7 \mathrm{U} / \mathrm{ml}$ respectively, $p<0.001)$.

There was strong and moderate positive correlation between RR achivement and aCCP-negativity $(r=0.57, p<0.05)$, low aCCP level $(r=0.45, p<0.05)$ and $\triangle D A S 28$ $(r=0.35, p<0.05)$.

Results of multinomial logistic regression analyses (SPSS, V.22, IBM) showed that RF-negativity was the only independent predictor of $R R$ achievement $(B=3.14$, $p<0.05)$.

$41.7 \%$ pts in RR achieved clinical remission by DAS28 as well. Only $19.7 \%$ pts achieved clinical remission without $R R(p<0.05$ vs pts achieved clinical and $R R)$ and $12.5 \%$ pts achieved RR without clinical response (DAS28 $>5.1, \triangle \mathrm{DAS} 28 \leq 1.2$ or any value of DAS28 with $\triangle \mathrm{DAS} 28<0.6 ; \mathrm{p}<0.01)$.

After 2 years of treatment the mean value of $\triangle \mathrm{DAS} 28$ in comparison groups (with and without $R R$ ) did not differ $(2.79 \pm 0.28$ vs $2.33 \pm 0.17$ respectively) so there was a discrepancy between clinical and radiological outcome.

Conclusions: Baseline predictors associated with achieving of radiographic remission in pts with RA are RF- and aCCP-negativity, low level of aCCP, but independent predictor of radiographic remission achievement is the only RF-negativity.

The discrepancy between the frequency of clinical and radiographic remission achievement in RA patients is observed.

After 2 yrs of non-biological DMARDs treatment $41.7 \%$ pts with RA achieves radiographic and clinical remission at the same time.

Disclosure of Interest: None declared

DOI: 10.1136/annrheumdis-2017-eular.2682

\section{THU0095 IMPACT OF ULTRASOUND-DETECTED TENOSYNOVITIS AND SYNOVITIS ON DISEASE FLARE, DISABILITY AND RADIOGRAPHIC PROGRESSION IN PATIENTS WITH RA IN CLINICAL REMISSION: RESULTS OF THE STARTER STUDY}

G. Sakellariou ${ }^{1}$, G. Filippou ${ }^{2}$, E. Bellis ${ }^{3}$, C.A. Scirè ${ }^{4}$, G. Carrara ${ }^{5}$, A. Iagnocco $^{6}$ on behalf of STARTER study group and US Study Group of the Italian Society for Rheumatology. ${ }^{1}$ IRCCS Policlinico San Matteo Foundation, Pavia; ${ }^{2}$ University of Siena, Siena; ${ }^{3}$ Ospedale Mauriziano, Torino; ${ }^{4}$ Italian Society for Rheumatology, Milan; ${ }^{5}$ Italian Society for Rheumatology, Milano; ${ }^{6}$ Università degli Studi di Torino, Torino, Italy

Background: In patients with RA in remission subclinical ultrasonographic (US) synovitis (-S) relates to flare and radiographic progression. The impact of tenosynovitis (-T) on flare, disability and radiographic progression is not known.

Objectives: To evaluate the predictive role of US-detected tenosynovitis and synovitis in RA patients in remission on flare, disability and radiographic progression over 12-months.

Methods: STARTER is a multicentre cohort study of the US Study Group of the Italian Society for Rheumatology. Participants were selected on the basis of a reliability exercise and the availability of high-end equipment with high frequencies probes. Patients with RA in remission underwent clinical and US evaluation. US -T and -S were assessed categorically by Grey Scale (GS) and power Doppler (PD) at 11 joints, extensor and flexor tendons in both hands. Patients were assesed at baseline, 6 and 12 months. The primary outcome was flare within 12 months (defined as increase in DAS28 $>1.2$ or $>0.6$ if final DAS28 $>3.2$ ). The secondary outcomes were progression of disability (increase $\geq 2.3$ in the Health Assessment Questionnaire (HAQ)) and radiographic progression (increase in the total Sharp van Der Hejide score (SHS) $\geq 4.3$ ) at 12 months. Logistic models were used to

Table 1

\begin{tabular}{lcc}
\hline & OR $(95 \% \mathrm{Cl})$ & Adj OR $(95 \% \mathrm{Cl})$ \\
\hline$\Delta$ DAS28 $>1.2$ or $>0.6$ if final & \\
PDS $28>3.2$ & \\
PD-S & $0.59(0.16,2.15)$ & $0.47(0.12,1.82)$ \\
PD-T + PD-S & $1.64(0.93,2.90)$ & $1.59(0.86,2.92)$ \\
GS-T & $2.75(1.45,5.20)$ & $2.09(1.06,4.13)$ \\
GS-S & $1.59(0.53,4.72)$ & $1.37(0.42,4.41)$ \\
GS-T + GS-S & $2.18(0.97,4.92)$ & $1.88(0.79,4.46)$ \\
$\Delta$ HAQ $\geq 0.23$ & $2.88(1.34,6.14)$ & $2.25(1.00,4.06)$ \\
PD-T & & \\
PD-S & $0.64(0.13,3.03)$ & $0.81(0.16,4.01)$ \\
PD-T + PD-S & $0.79(0.37,1.68)$ & $0.71(0.32,1.54)$ \\
GS-T & $1.49(0.68,3.26)$ & $1.33(0.57,3.11)$ \\
GS-S & $0.77(0.22,2.73)$ & $0.64(0.17,2.45)$ \\
GS-T + GS-S & $0.83(0.34,1.97)$ & $0.61(0.24,1.54)$ \\
$\Delta$ Total SHS $\geq 4.3$ & $0.93(0.42,2.02)$ & $0.64(0.27,1.53)$ \\
PD-T & & \\
PD-S & $0.82(0.09,7.37)$ & $0.54(0.05,5.76)$ \\
PD-T + PD-S & $1.61(0.71,3.64)$ & $1.60(0.68,3.79)$ \\
GS-T & $1.53(0.60,3.93)$ & $1.09(0.38,3.11)$ \\
GS-S & $0.78(0.08,7.88)$ & $0.67(0.06,7.50)$ \\
GS-T + GS-S & $2.38(0.72,7.84)$ & $1.78(0.49,6.48)$ \\
\hline
\end{tabular}

measure the relationship between GS-T/-S, PD-T/-S and outcomes, results were presented as odds ratios (OR) and 95\% confidence interval $(\mathrm{Cl})$, adjusted for pre-specified confounders.

Results: 361 patients $(72.3 \%$ f, mean age (sd) 56.1 (13.3), median disease duration (IQR) 7.1 years (3.6-13.5)) were included, complete radiographs were available for $189 / 361$ (52.3\%) patients. 98/326 (30.6\%) patients had a flare within 12 months, 70/340 (20.59\%) had an increase in HAQ and 39/189 (20.6\%) radiographic progression. Results are presented in Table 1. Flare was predicted only if $-T$ and $-S$, assessed by both GS and PD, were concurrently present, while both $-T$ and-S and their combination did not predict $\mathrm{HAQ}$ or SHS progression.

Conclusions: In patients with RA in clinical remission, US-detected synovial and tenosynovial inflammation relates to the risk of flare, while in a short term follow-up the effect on disability and radiographic progression is limited. These results might have been influenced by the short follow-up and limited power for secondary outcomes. US might integrate the clinical management of RA patients in clinical remission.

Disclosure of Interest: None declared

DOI: 10.1136/annrheumdis-2017-eular.4774

\section{THU0096 MULTI-BIOMARKER DISEASE ACTIVITY (MBDA) IS ASSOCIATED WITH THE PROGRESSION OF STRUCTURAL BONE DAMAGE IN RHEUMATOID ARTHRITIS PATIENTS IN REMISSION}

G. Schett ${ }^{1}$, S. Finzel ${ }^{2}$, M. Hagen ${ }^{1}$, M. Englbrecht ${ }^{1}$, J. Haschka ${ }^{3}$,

C. Figuereido ${ }^{4}$, J. Fogagnolo Cobra ${ }^{4}$, J. Rech ${ }^{1}$. ${ }^{1}$ University of

Erlangen-Nuremberg, Erlangen; ${ }^{2}$ University Medical Center Freiburg,

Rheumatology and Clinical Immunology, Freiburg, Germany; ${ }^{3}$ St.Vincent

Hospital, Vinforce Study Group, Medical University of Vienna, Vienna, Austria;

${ }^{4}$ Institutio de Rheumatologia, Sao Paolo, Brazil

Background: Due to an increasing number and an earlier use of effective disease modifying anti-rheumatic drug (DMARD) therapy a growing number of rheumatoid arthritis (RA) patients reaches a state of clinical remission of disease. Whether clinical remission completely protects from structural bone damage, however, is still a matter of debate as conventional radiographic methods have their limitation in the sensitivity to characterize bone damage. Furthermore, residual activity of inflammation associated with the generation of inflammatory markers may characterize a subset of RA patients in remission, associated with higher prevalence and/or progression of bone damage.

Objectives: To test whether residual systemic inflammation is associated with structural bone damage and the progression of structural bone damage in RA patients in sustained remission.

Methods: RA patients (i) fulfilling the 2010 EULAR/ACR classification criteria of RA, (ii) having a positive anti-citrullinated protein antibody (ACPA) status and (iii) being in DAS28-ESR remission for 6 months were included. High-resolution peripheral quantitative computed tomography (HR-pQCT) of the right hand was done at baseline and after 1year. Erosion numbers and erosion volumes were assessed in the metacarpophalangeal joints. Vectra-DA tests measuring the serum levels of twelve different inflammation markers (CRP, SAA, IL-6, TNFR1, MMP-1, MMP-3, EGF, VEGF-A, VCAM-1, YKL-40, leptin and resistin) were performed in the baseline samples. MBDA score was calculated according to previously defined algorithms with low MDBA score defined as $<30$ units and moderate to high scores as $\geq 30$ units (1).

Results: $100 \mathrm{ACPA}+\mathrm{RA}$ patients in sustained remission were investigate (mean \pm SD age: $57 \pm 14$ ys; disease duration: $4.8 \pm 4.9$ ys; DAS28: $1.7 \pm 0.5 ; 63 \%$ females; $100 \%$ MTX treatment, $38 \%$ MTX+TNFi treatment). 65 patients had low $(<30), 25$ patients has moderate $(>30-44$ and 10 patients had high $(>44)$ MBDA scores. Patients in the different MBDA categories had similar age, sex, disease duration, DAS28 scores and DMARD treatment. Baseline HR-pQCT analysis showed that erosion numbers and volumes were significantly $(\mathrm{p}<0.001)$ higher in patients with high MBDA scores. Higher erosion numbers $(>10)$ and larger erosions $\left(>10 \mathrm{~mm}^{3}\right)$ were exclusively found in patients with moderate to high MBDA scores. MBDA scores were correlated to erosion numbers $(p<0.001)$ and volumes $(p=0.0018)$. Longitudinal analysis showed significant progression of erosions only in patients with high MBDA scores. Furthermore, progression $\left(>5 \mathrm{~mm}^{3}\right.$ increase in volume) over 1 year was confined to methotrexate treated patients, while tumor necrosis factor inhibitor treated patients were protected from progression even in case of moderate to high MBDA scores. However there were only 4 patients treated with TNFi that had a high MBDA score.

Conclusions: These data show that residual disease activity assessed by MBDA score is associated with structural damage and progression of structural damage in RA patients in sustained remission.

\section{References:}

[1] Curtis JR, van der Helm-van Mil AH, Knevel R, et al.Validation of a novel multi-biomarker test to assess rheumatoid arthritis disease activity. Arthritis Care Res 2012;64:1794-803.

Disclosure of Interest: None declared

DOI: 10.1136/annrheumdis-2017-eular.6227 